\title{
Digital Capital
}

Sora Park. Palgrave Macmillan. London, 2017, 247 páginas. ISBN: 978- 1- 137- 59331- 3.

El libro Digital Capital de Sora Park se propone describir y comprender los diversos factores que incidirían en el comportamiento de los sujetos hacia las tecnologías digitales y cómo dichas acciones podrían relacionarse con el desarrollo de una sociedad digital. Para profundizar en este fenómeno la autora utiliza un consolidado cuerpo de literatura, sobre alfabetización e inclusión digital, que le permite dar cuenta del resultado -más o menos efectivo- de distintas experiencias internacionales relacionadas con esta temática. Específicamente, para referirse a la relación entre los sujetos y las tecnologías, Park aborda el concepto de capital digital definido como un conjunto predeterminado de disposiciones que influyen en cómo las personas se involucran con la tecnología digital. En este sentido, logra posicionar este nuevo capital junto a las nociones ya conocidas de capital cultural, capital social y capital económico presentes en el trabajo de Bourdieu (2001)․ El libro se estructura en 10 capítulos; el primero corresponde a la introducción y los 9 restantes se organizan en tres grandes secciones: (i) Re-conceptualización de la inclusión digital, donde se profundiza el concepto de capital digital para una comprensión más matizada de la inclusión digital; (ii) Nuevos problemas y soluciones, donde se examina el estado actual de la sociedad digital y los tipos de habilidades que se requerirían para prosperar en un entorno digital y (iii) El cambio de paradigma en las políticas de inclusión digital, donde se proponen nuevos marcos de acción para la formación digital de los individuos y para el desarrollo de políticas relacionadas con la inclusión digital.

La primera sección del libro comprende los capítulos 2, 3, 4 y 5. A partir del capítulo 2 se abordan los diversos factores que influirían en la manera en que las personas adoptan y se adaptan a las tecnologías. Aquí se menciona la importancia de considerar elementos contextuales, sociales y personales. A pesar de la identificación de estos factores, no resulta del todo claro cuáles serían las condiciones mínimas que debiesen satisfacerse para lograr un uso eficiente de las tecnologías. Ahora bien, se mencionan dos conceptos que permitirán profundizar en la relación del individuo con las tecnologías; por una parte, se plantea la idea de involucramiento digital y, por otra, la de segregación digital. Para Park, abordar el involucramiento y la segregación digital significa considerar la importancia de las condiciones previas de los individuos que delimitan su disposición y preparación frente a las tecnologías y, con esto, sus posibilidades de movilizar conocimientos para obtener beneficios en un futuro. La autora propone, en este punto, que cada persona habita un ecosistema tecnológico lo que incide en las problemáticas que pueden emerger en el uso que hace de las tecnologías

1 Bourdieu, P. (2001). Poder, derecho y clases sociales. En Palimpsesto (Vol. 6). 
digitales. A continuación, en el capítulo 3, se profundiza en el concepto de segregación o exclusión digital como una nueva forma de desigualdad, presente en nuestras sociedades, que se originaría por un uso diferenciado de los recursos digitales. Se argumenta que este nuevo tipo de desigualdad tendría consecuencias a largo plazo al promover la exclusión de nuevos grupos sociales. Así, dentro de las nuevas formas de exclusión digital se destacarían: i) la privatización digital relativa, asociada a la percepción negativa de los usuarios que poseen bajo nivel de uso de internet en relación con la sociedad digital que los rodea y ii) el acceso interrumpido asociado al bajo nivel de involucramiento de aparataje técnicotecnológico que obstaculizaría la adquisición y desarrollo de habilidades requeridas por parte de los usuarios. En el capítulo 4, Park profundizará mayormente en la idea de capital digital diferenciándolo de otras formas de capital como el técnico y el tecnológico (Carlson \& Isaacs, 2018; Grigoriev, Yeleneva, Golovenchenko, \& Andreev, 2014)². La autora propondrá una crítica a estas dos formas de capital ya que no consideran los conocimientos previos de los usuarios en su acceso a las tecnologías. Para Park una nueva definición de capital digital permitirá tener una perspectiva más amplia de las tecnologías al enfatizar en la importancia de desarrollar habilidades que sirvan para: (i) usar eficientemente las tecnologías y (ii) lograr movilizar recursos disponibles para obtener beneficios futuros. Esta primera sección del libro culmina con el capítulo 5 que expone los principales elementos del capital digital que influirán en la adopción y uso de tecnologías por parte del usuario. Estos elementos referirán a: (i) infraestructura, (ii) calidad, (iii) accesibilidad, (iv) habilidades, (v) capacidad técnica, (vi) actitud del usuario y (vii) habilitadores sociales. Así, el libro propone que las condiciones preexistentes que permitirán u obstaculizarán la adaptación a nuevas tecnologías digitales tendrán que entenderse dentro de un contexto global que considere, a su vez, al capital económico, cultural y social.

La segunda sección de este libro, que considera los capítulos 6, 7 y 8, ahonda en la existencia de la brecha digital entendida como la desigualdad que existe entre las personas respecto del acceso a las nuevas tecnologías. A partir del capítulo 6, la autora se propone enriquecer el concepto de infraestructura, yendo más allá de la mera conectividad, al redefinirlo como el acceso que tiene un usuario al ecosistema global de tecnología digital. En este sentido, la infraestructura constituirá una problemática mundial que habrá que considerar en relación con la segregación digital de los usuarios. Aquí se argumenta la importancia que tiene considerar el logro digital de los estudiantes en sus contextos educativos a partir de la consideración de su acceso a internet, lo que desencadenará en consecuentes procesos de exclusión/segregación social. Disminuir la brecha digital entre usuarios que tienen acceso a

2 Carlson, A., \& Isaacs, A. M. (2018). Technological capital: an alternative to the digital divide. Journal of Applied Communication Research, 46(2), 243-265. https://doi.org/10.1080/00909882.2018.1437279

Grigoriev, S. N., Yeleneva, J. Y., Golovenchenko, A. A., \& Andreev, V. N. (2014). Technological capital: A criterion of innovative development and an object of transfer in the modern economy. Procedia CIRP, 20(C), 56-61. https:// doi.org/10.1016/j.procir.2014.06.144 
internet de los que no lo tienen, continuará siendo un problema persistente a nivel global. En este escenario se menciona la existencia de elementos comunes que estarían asociados a la inclusión digital a partir de la consideración de cuatro contextos: Estados Unidos, Unión Europea, Australia y Sudáfrica. A partir de la realidad de estos 4 territorios se identifica que la calidad del acceso a internet, a las actividades online y las habilidades de los usuarios estarán estrechamente relacionadas con la brecha digital. Finalmente, la autora propone que el primer paso para esclarecer el problema de la segregación digital radicaría en una reflexión sobre qué tan integradas se encuentran las tecnologías digitales a cada uno de los aspectos de la vida diaria de los sujetos, entendiendo que esta situación, ineludiblemente, se incrementará con el paso de los años y el avance de la tecnología. En el capítulo 7 a partir de la alfabetización digital se evidencia la importancia de profundizar en aquellas habilidades que deberían desarrollar los usuarios para poder navegar y comunicarse en el espacio online de manera eficiente. Aquí la fluidez digital entendida como la encapsulación de una variedad de habilidades necesarias para que los sujetos puedan vivir en un entorno digital que evoluciona constantemente, resultará igualmente decisiva. Este último punto se retoma en el capítulo 8 donde se argumenta que la fluidez digital representaría un nivel más alto de involucramiento digital y, en este sentido, para promoverla sería necesario que los sujetos pudieran desarrollar ciertos elementos como conocimientos técnicos, confianza digital, creación de redes y fluidez de la información. Este último elemento se destaca al evidenciarse que una de las habilidades esenciales en la era actual corresponde a la capacidad de poder convertir datos en información valiosa. Lo anterior se traducirá en la capacidad de acceder a grandes cantidades de datos a partir del uso de habilidades necesarias para la extracción y levantamiento de información útil. A partir de un estudio en el Reino Unido, Park señala que es posible distinguir diversas habilidades digitales, básicas y generales, que considerarían los siguientes elementos: (i) manejo de la información, (ii) comunicación, (iii) transacciones (compra-venta de bienes y servicios y organización financiera, entre otros), (iv) resolución de problemas y (v) creación. Aquí, se concluye que la capacidad de compartir o de poder convertirse en receptores activos de información constituirá una habilidad mínima para prosperar dentro de un entorno digital y, con esto, una posibilidad real de participación digital efectiva.

Finalmente, ahondando en la tercera sección, compuesta por los capítulos 9 y 10, se profundiza en la importancia de considerar la alfabetización digital más allá de una habilidad técnica para pasar a considerarla como una capacidad amplia que permite, a partir del uso de tecnologías digitales, una mayor participación económica y social. A partir de esta premisa, la autora sugiere nuevas propuestas que podrían incidir en un replanteamiento de las políticas e intervenciones relacionadas con la inclusión digital. De manera particular, en el capítulo 9 , se intenta esclarecer la relación entre la fluidez digital y el nivel de capital digital de un usuario, de manera tal de poder comprender por qué algunos usuarios aprenden a utilizar tecnologías digitales de forma más rápida que otros. En esa línea el libro evidencia, a partir 
de la observación de diversos usuarios novatos con distintos grados de capital digital, que existirían diferentes métodos o modelos de alfabetización/capacitación que permitirían ilustrar cómo los usuarios aprenden a usar tecnologías. Para ello, Park considera dos ejes que le permiten agrupar los diferentes métodos de capacitación digital existentes. Aquí se menciona el eje entrenamiento/apoyo y el eje formal/informal. Las combinaciones de estos ejes permiten definir cuatro métodos de capacitación: (1) entrenamientos en entornos formales, (2) entrenamientos en entornos informales que se caracterizan por suscitar aprendizajes estructurados entre pares, (3) capacitaciones formales con un sistema de soporte integrado, mediante el uso de intermediarios y (4) capacitaciones informales a través de personas no expertas. Sin embargo, es preciso considerar que las observaciones que han permitido evidenciar los métodos utilizados, para entregar un entrenamiento a los usuarios, surgen o han sido levantadas en contextos de países desarrollados sin dar mayores luces sobre los problemas que podrían emerger al considerar contextos de mayor vulnerabilidad o la realidad de muchos países latinoamericanos en que el acceso a las tecnologías depende, en gran medida, del grupo socioeconómico al que pertenece el usuario. Finalmente, el capítulo culmina refiriéndose a la fluidez digital que constituirá una habilidad requerida para poder adaptarse a las nuevas tecnologías que eventualmente emergerán en el futuro. La autora sostiene que dicha adaptación necesariamente requerirá un “desaprendizaje” (unlearning) del usuario al tener que renunciar a los conocimientos, valores, creencias o prácticas que ya tenía para poder adaptarse a las innovaciones y a los entornos tecnológicos que se encuentran en constante cambio.

Por último, en el capítulo 10 se realiza un análisis en torno a las políticas que se han fomentado en países desarrollados en materias de brecha digital argumentando que, si bien, centrarse en la infraestructura resulta relevante para reducir la brecha digital, esta preocupación solo constituirá una condición previa para la inclusión digital. El libro problematiza, en este sentido, que el simple acceso a las tecnologías no se traduce automáticamente en resultados favorables en su uso. En relación con lo anterior la autora cuestiona que uno de los principales focos de la política haya sido centrarse en la inclusión digital desatendiendo el desafío que emerge al considerar la constante evolución y desarrollo de las tecnologías. La reflexión finaliza acudiendo a las contribuciones de van Dijk (2006), quien delimita un marco conceptual que permite atender y responder a las desigualdades creadas por la tecnología. En concreto, se expone que las desigualdades digitales proveerían distintos tipos de desigualdad que podrían incluso incidir en desigualdades tecnológicas, inmateriales, materiales, sociales y educativas de los individuos. De esta manera, se argumenta que el objetivo último de las políticas y los programas que se dedican al ámbito digital no debiera orientarse a que las personas utilicen la tecnología de manera uniforme, sino que debieran considerar el ecosistema tecnológico digital al que pertenece el usuario de modo

van Dijk, J. A. G. M. (2006). Digital divide research, achievements and shortcomings. Poetics, 34(4-5), 221-235. https://doi.org/10.1016/j.poetic.2006.05.004 
que las personas puedan utilizar y acceder a la tecnología considerando la diversidad de sus contextos y los recursos disponibles para su beneficio. Aquí resulta importante que las políticas abocadas a la capacitación tecnológica puedan garantizar y focalizarse en las personas que ya se encuentran excluidas, de forma que el acceso a las tecnologías no se traduzca en una doble exclusión. Esto sugiere que las tecnologías no emerjan como un nuevo elemento de segregación social para personas que ya se encuentran en desventaja, sobre todo, al tener presente que las necesidades de los usuarios son diferentes y contextuales.

La postura epistemológica que moviliza a Park se basa en la comprensión de las tecnologías como herramientas que posibilitarían el cumplimiento de propósitos tangibles. Si bien su noción de capital digital contribuye a la consideración de las características previas de los sujetos a la hora de comprender su relación con la tecnología, a lo largo del texto existe una visión marcadamente técnica de estas tecnologías. Esta perspectiva se distanciaría de otros paradigmas que enfatizan en la relación e influencia mutua que existiría entre los sujetos y las tecnologías. En este sentido, enriquecer la comprensión de las tecnologías a partir de nuevas perspectivas emergentes como las postcualitativas permitiría reconocer la agencia intrínseca que poseen las tecnologías que, alejándose de toda pretensión de neutralidad, modificarán y adaptarán a quien las crea y utiliza (Barad, 2014; Beach, Bagley, \& Marques, 2018) ${ }^{4}$. Considerar estas perspectivas permitiría enriquecer la propuesta de la autora, poniendo como foco de atención la relación del individuo y la tecnología y no solo la capacidad del individuo de poder acceder y adaptarse a las nuevas tecnologías.

\author{
DANY LÓPEZ GonZÁLEZ \\ Facultad de Educación de la Pontificia \\ Universidad Católica de Chile \\ CANDidato a Doctor en Educación
}

\author{
Macarena Salas Aguayo \\ Facultad de Educación de la Pontificia \\ Universidad Católica de Chile \\ CANDidata a Doctora en Educación
}

4 Barad, K. (2014). Diffracting Diffraction: Cutting Together-Apart. Parallax, 20(3), 168-187. https://doi.org/10.10 80/13534645.2014.927623

Beach, D., Bagley, C., \& Marques, S. (2018). Agential Realism and Educational Ethnography The Return to Ontology. En The Wiley Handbook of Ethnography of Education (pp. 403-421). John Wiley \& Sons, Inc. 